\title{
The changing epidemiology of bacillary dysentery and characteristics of antimicrobial resistance of Shigella isolated in China from 2004-2014
}

Zhaorui Chang, Jing Zhang, Lu Ran, Junling Sun, Fengfeng Liu, Li Luo, Lingjia Zeng, Liping Wang, Zhongjie Li, Hongjie Yu and Qiaohong Liao*

\begin{abstract}
Background: Bacillary dysentery caused by bacteria of the genus Shigella is a significant public health problem in developing countries such as China. The objective of this study was to analyze the epidemiological pattern of bacillary dysentery, the diversity of the causative agent, and the antimicrobial resistance patterns of Shigella spp. for the purpose of determining the most effective allocation of resources and prioritization of interventions.

Methods: Surveillance data were acquired from the National Infectious Disease Information Reporting System (2004-2014) and from the sentinel hospital-based surveillance system (2005-2014). We analyzed the spatial and temporal distribution of bacillary dysentery, age and sex distribution, species diversity, and antimicrobial resistance patterns of Shigella spp.

Results: The surveillance registry included over 3 million probable cases of bacillary dysentery during the period 2004-2014. The annual incidence rate of bacillary dysentery decreased from 38.03 cases per 100,000 person-years in 2004 to 11.24 cases per 100,000 person-years in 2014. The case-fatality rate decreased from $0.028 \%$ in 2004 to 0. $003 \%$ in 2014. Children aged $<1$ year and 1-4 years were most affected, with higher incidence rates (228.59 cases per 100,000 person-years and 92.58 cases per 100,000 person-years respectively). The annual epidemic season occurred between June and September. A higher incidence rate of bacillary dysentery was found in the Northwest region, Beijing and Tianjin during the study period. Shigella flexneri was the most prevalent species that caused bacillary dysentery in China (63.86\%), followed by Shigella sonnei (34.89\%). Shigella isolates were highly resistant to nalidixic acid (89.13\%), ampicillin (88.90\%), tetracycline (88.43\%), and sulfamethoxazole (82.92\%). During the study period, isolates resistant to ciprofloxacin and cefotaxime increased from 8.53 and 7.87\% in 2005 to 44.65 and 29. $94 \%$ in 2014 , respectively.
\end{abstract}

Conclusions: The incidence rate of bacillary dysentery has undergone an obvious decrease from 2004 to 2014. Priority interventions should be delivered to populations in northwest China and to individuals aged $<5$ years. Antimicrobial resistance of Shigella is a serious public health problem and it is important to consider the susceptibility profile of isolates before determining treatment.

Keywords: Epidemiology, Bacillary dysentery, Resistance, Shigella

\footnotetext{
* Correspondence: liaogh@chinacdc.cn

Division of Infectious Diseases, Key Laboratory of Surveillance and

Early-warning on Infectious Disease, Chinese Center for Disease Control and Prevention, 155 Changbai Road, Changping District, Beijing 102206, People's Republic of China
} 


\section{Background}

Bacillary dysentery, which is primarily transmitted by the fecal-oral route via contaminated food, water, or person-to-person contact [1-5], is an important enteric infectious disease caused by Shigella spp. The major symptoms of bacillary dysentery include diarrhea, fever, abdominal pains, tenesmus and stool with blood or mucus [6]. Annually, there are 165 million reported or confirmed cases of bacillary dysentery and 1.1 million deaths worldwide, predominantly in developing countries [7]. In mainland China, the annual morbidity and mortality of bacillary dysentery ranked in the top ten of 39 notifiable infectious diseases from 2004 to 2014.

Bacillary dysentery can be caused by four Shigella species: S. dysenteriae, S. flexneri, S. boydii, and S. sonnei [8]. Shigella sonnei is the most prevalent Shigella species in developed countries. In developing countries, S. flexneri tends to be predominant, while $S$. dysenteriae and $S$. boydii are comparatively rare [7]. With the overuse of antibiotics, antimicrobial resistance has been increasing among Shigella isolates in recent years, limiting the possibilities for suitable empirical antibiotic treatments [9]. Knowledge of the disease burden and epidemiological characteristics of bacillary dysentery is helpful for allocating resources and prioritizing interventions. Therefore, in this study, we investigated the temporal and spatial distribution of bacillary dysentery, determined the high-risk populations of bacillary dysentery, and determined the predominant circulating species and antimicrobial resistance patterns of Shigella based on existing data sources from the National Infectious Disease Information Reporting System (NIDRS) from 2004 to 2014 and the sentinel-based bacillary dysentery surveillance system from 2005 to 2014 in China.

\section{Methods}

\section{National surveillance for bacillary dysentery}

Bacillary dysentery was added to the list of notifiable diseases in China in 1956. From 1956 to 2003, the number of cases and deaths by province were reported monthly to the Chinese Center for Disease Control and Prevention (China CDC). After 2004, the NIDRS, an internet-based notifiable infectious disease reporting system, was established [10]. Thereafter, all probable and confirmed cases along with individual data were reported online to the China CDC by clinicians within 24 $\mathrm{h}$ of diagnosis. Clinicians diagnose bacillary dysentery cases according to the unified diagnosis criteria issued by the Chinese Ministry of Health. A probable case of bacillary dysentery was defined as a patient with the following clinical features: fever, chills, abdominal pain, tenesmus, bloody or mucus stool or stool containing $>15 /$ high power field (HPF) leukocytes or purulent cells, and microscopically discernible red blood cells and phagocytic cells. A confirmed case was defined as a patient with Shigella spp. isolated from a stool specimen [11]. The individual data includes gender, date of birth, address, case classification (probable or confirmed), date of onset, and date of death (if applicable). All data used in this study for bacillary dysentery cases reported from 1 January 2004 to 31 December 2014 in China were acquired from the NIDRS.

\section{Sentinel hospital-based bacillary dysentery surveillance}

Sentinel hospital-based bacillary dysentery surveillance was established in 2005 to monitor the predominant circulating species and antimicrobial resistance patterns of Shigella in China. The surveillance system consists of 20 sentinel hospitals distributed in Beijing, Gansu, Qinghai, Shanxi, Henan, Heilongjiang, Anhui, Fujian, Guizhou, and Shanghai. This coverage represents variation in geographical features, economic development, and sanitary conditions. A national surveillance protocol and laboratory testing assays were developed by the China CDC and are used by all sentinel sites [12].

\section{Specimen collection and testing}

In each sentinel hospital, fecal specimens were collected from patients with diarrhea and clinically suspected dysentery who had not been given daily antimicrobial treatment. Fresh fecal samples were inoculated in Cary-Blair medium (most frequently, Qingdao Hope Bio-Technology Co., Ltd, Shandong, China) and sent to the regional CDC laboratory within $4 \mathrm{~h}$. Stool samples were streaked onto XLD agar and SS agar and then incubated at $37{ }^{\circ} \mathrm{C}$ for 18-24 h. Colorless and transparent colonies were screened using triple sugar iron agar and motility indoleurea agar. Presumptive positive colonies were then confirmed using API 20E strips (bioMérieux, Marcy l'Etoile, France). All confirmed isolates were serotyped using commercial antisera (Denka Seiken Co. Ltd., Tokyo, Japan).

More than 300 fecal specimens were collected at each sentinel hospital every year, among which at least 30 specimens were selected for isolation and identification of bacteria each month in the epidemic season (May-October) and 10 specimens in the non-epidemic season (November-April). Sampling was designed to ensure that $>50 \%$ of samples selected for isolation and identification of bacteria were from children.

\section{Antimicrobial susceptibility}

The ability of different Shigella isolates to resist the inhibitory effects of different antibiotics was tested by the provincial CDCs. At least 30\% of the confirmed Shigella isolates, which cover different species, were selected for antimicrobial susceptibility testing each month. Minimal inhibitory concentrations of the following nine antimicrobial agents were determined using the agar 
dilution method according to the Clinical and Laboratory Standards Institute (CLSI) guidelines [13]. As such, ampicillin, amoxicillin, cefotaxime, cephalothin, gentamicin, nalidixic acid, ciprofloxacin, tetracycline, sulfamethoxazole. Escherichia coli (American Type Culture Collection strain 25922) was used for quality control. Susceptible and non-susceptible isolates were identified according to the criteria used for enterobacteria as suggested by the CLSI.

\section{Data analysis}

We included cases with illness onset reported to the NIDRS from 1 January 2004 to 31 December 2014 in the analysis. We not only calculated a crude incidence rate (number of cases divided by the corresponding population), a case-fatality rate (number of deaths divided by the number of probable and confirmed cases), and age-specific rates by sex (number of cases occurring in a specific age group divided by the corresponding population for each sex), but we also determined the seasonal patterns and geographic distribution of bacillary dysentery. Population data for the study period were extracted from the National Bureau of Statistics of China [14]. In addition, we analyzed the proportions of Shigella spp. isolated from laboratory-confirmed cases of bacillary dysentery and the resistance rates of Shigella collected from sentinel hospitals during 2005-2014. The Cochran-Armitage trend test was used to examine the temporal trends in the annual morbidity of bacillary dysentery, the proportion of bacillary dysentery cases by age groups, and the antimicrobial resistance of Shigella isolates. The index $Z>0$ denotes an increasing trend in the annual morbidity of bacillary dysentery, the proportion of bacillary dysentery cases by age groups, and the antimicrobial resistance of Shigella isolates, whereas $Z<0$ denotes a decreasing trend. The trend was considered to be significant when $\mathrm{P}$ was $<0.05$. A chi-square test was used to examine whether the sex-specific incidence in children younger than 5 years was significantly different between male and female individuals, with a significance level of $\alpha=0.05$. We conducted all analyses with SAS 9.4 (SAS Institute Inc., Cary, USA).

We used a seasonal index to understand seasonal patterns of bacillary dysentery in China. This index was calculated by month as the average case count for the given month divided by the average monthly case count during the entire 11 years of surveillance from 2004 to 2014 [15]. No obvious seasonal pattern was expected if the seasonal index of each month was close to 1.0 [15].

The incidence rate of three time periods by province was used to demonstrate the geographic distribution of bacillary dysentery. The three time periods (2004-2006, 2007-2009, 2010-2014) were categorized according to the average incidence of bacillary dysentery $(>30$ per 100,000 person-years, $20-30$ per 100,000 person-years and $<20$ per 100,000 person-years) in China. The software ArcGIS version 10.0 (ESRI, Redlands, CA, USA) was used to describe the spatial distribution of bacillary dysentery using a county-level polygon map.

\section{Results}

Temporal trend in incidence rate and case-fatality rate of bacillary dysentery

The reported annual incidence and case-fatality rate of bacillary dysentery from 2004 to 2014 according to the NIDRS are shown in Fig. 1. During 2004-2014, $3,342,847$ cases of bacillary dysentery were reported in China with an average incidence rate of 22.89 cases per 100,000 person-years. The annual incidence rate of bacillary dysentery decreased by approximately 3 -fold from 38.03 cases per 100,000 person-years in 2004 to 11.24 cases per 100,000 person-years in $2014(Z=-672.51$, $P<0.05$, by Cochran-Armitage trend test). During 20042014, a total of 636 deaths due to bacillary dysentery were

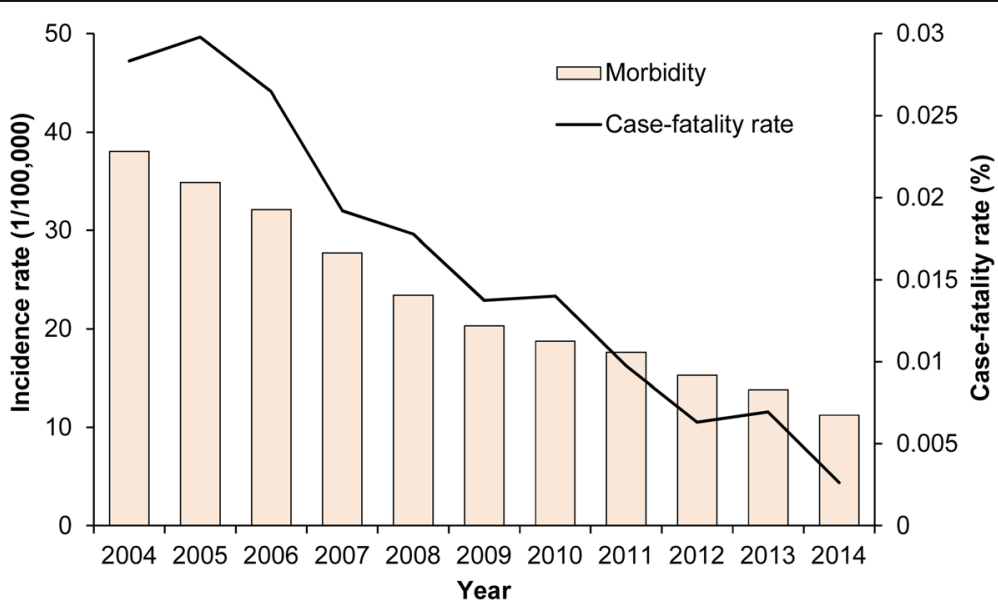

Fig. 1 Incidence rate and case-fatality rate of bacillary dysentery in China, 2004-2014 
reported, and the case-fatality rate of bacillary dysentery decreased from $0.028 \%$ in 2004 to $0.0026 \%$ in 2014 .

\section{Demographic features}

The incidence rate of bacillary dysentery varied greatly according to age groups, with the highest rate being 228.59 cases per 100,000 person-years observed in children $<1$ years of age, followed by an average incidence rate of 92.58 cases per 100,000 person-years in children $1-4$ years of age. Cases identified in children $<5$ years of age accounted for $31.47 \%$ of the total number of cases reported from 2004 to 2014. The data showed a male predominance in most age groups, with an overall male-to-female ratio of 1.31:1. Male predominance was most obvious within children $<5$ years of age, and the incidence of bacillary dysentery was 1.54 times higher in boys younger than 5 years than in girls of the same age (146.89 vs 93.46 cases per 100,000 person-years, $\chi^{2}$ $=46272.89, P<0.05$ ) (Fig. 2a). The proportion of bacillary dysentery cases that were identified in individuals $<5$ years of age or $\geq 60$ years of age increased from 2004 to 2014 ( $<5$ years of age: $27.85-33.77 \%$, CochranArmitage trend test: $Z=77.76, P<0.05 ; \geq 60$ years of age: $10.20-16.43 \%$, Cochran-Armitage trend test: $Z=$ 108.87, $P<0.05)$. In contrast, the proportion of cases identified in individuals $5-19$ years of age decreased

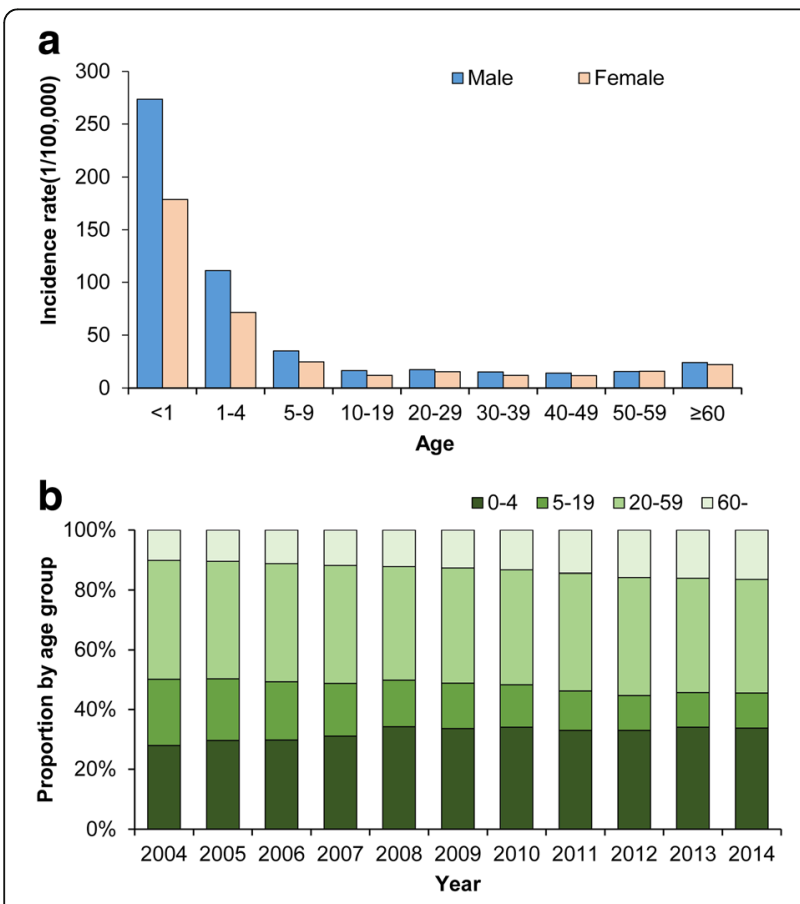

Fig. 2 Age and sex distribution of bacillary dysentery in China, 2004-2014. a The average annual incidence of bacillary dysentery by age and sex was calculated by dividing the total number of cases occurring in a specific age group during 2004-2014 by the corresponding population and multiplying by 100,000 . b The proportion of bacillary dysentery cases by age groups from $22.27 \%$ in 2004 to $11.73 \%$ in $2014(Z=-175.18, P$ $<0.05$, by Cochran-Armitage trend test) (Fig. 2b).

\section{Seasonal distribution}

In China during 2004-2014, bacillary dysentery presented obvious seasonal characteristic (Fig. 3a, b). The majority of bacillary dysentery cases occurred from June to September, during which $57.60 \%$ of cases were reported. The seasonal index was highest in August (1.80) when the average monthly incidence rate was 41.97 per 100,000 person-years.

\section{Geographical distribution}

Despite an overall decrease in incidence rate over the time period of the study, northwest China (Tibet, Xinjiang, Gansu, and Ningxia), Beijing, and Tianjin consistently had the highest incidence rates of bacillary dysentery from 2004 to 2014. Southeast China (Jiangsu, Shanghai, Fujian, Guangdong, and Hainan), northeast China (Jilin, Heilongjiang), and Inner Mongolia had relatively lower incidence rates of bacillary dysentery. The number of provinces with an incidence rate $>60$ per 100,000 person-years reduced from eight (Beijing, Ningxia, Tianjin, Tibet, Gansu, Xinjiang, Shannxi, and Guizhou) in 2004-2006 to two (Beijing and Tianjin) in 2010-2014. The number of provinces with an incidence rate $<20$ per 100,000 person-years progressively increased from five (Hainan, Inner Mongolia, Hunan, Guangdong, and Fujian) in 2004-2006 to 14 in 2007-2009 and to 21 in 2010-2014 (Fig. 4).

\section{Distribution of Shigella species that cause bacillary dysentery}

From 2005 to 2014, a total of 70,802 samples were collected and tested for the presence of Shigella bacteria in sentinel hospitals. From all tested samples, a total of 6278 Shigella isolates (8.87\%) were isolated. Of these, S. flexneri was the most prevalent (4009 isolates, 63.86\%), followed by S. sonnei (2191 isolates, 34.89\%). Shigella dysenteriae (38 isolates) and $S$. boydii (40 isolates) were relatively uncommon (only present in 2005, 2006, 2007 and 2011) and accounted for only 0.61 and $0.63 \%$ of the isolates, respectively (Fig. 5a).

Figure 5b shows the geographical distribution of Shigella species determined by serotyping isolates obtained from patients with diarrhea and clinically suspected dysentery during 2005-2014. Shigella flexneri was the most prevalent in central and northern China except for in Beijing; $S$. sonnei was the most prevalent in eastern and southern China (Shanghai, Guizhou and Fujian).

\section{Antimicrobial resistance of Shigella isolates}

The resistance rates of Shigella during the study period are shown in Table 1. Isolates had highest resistance 

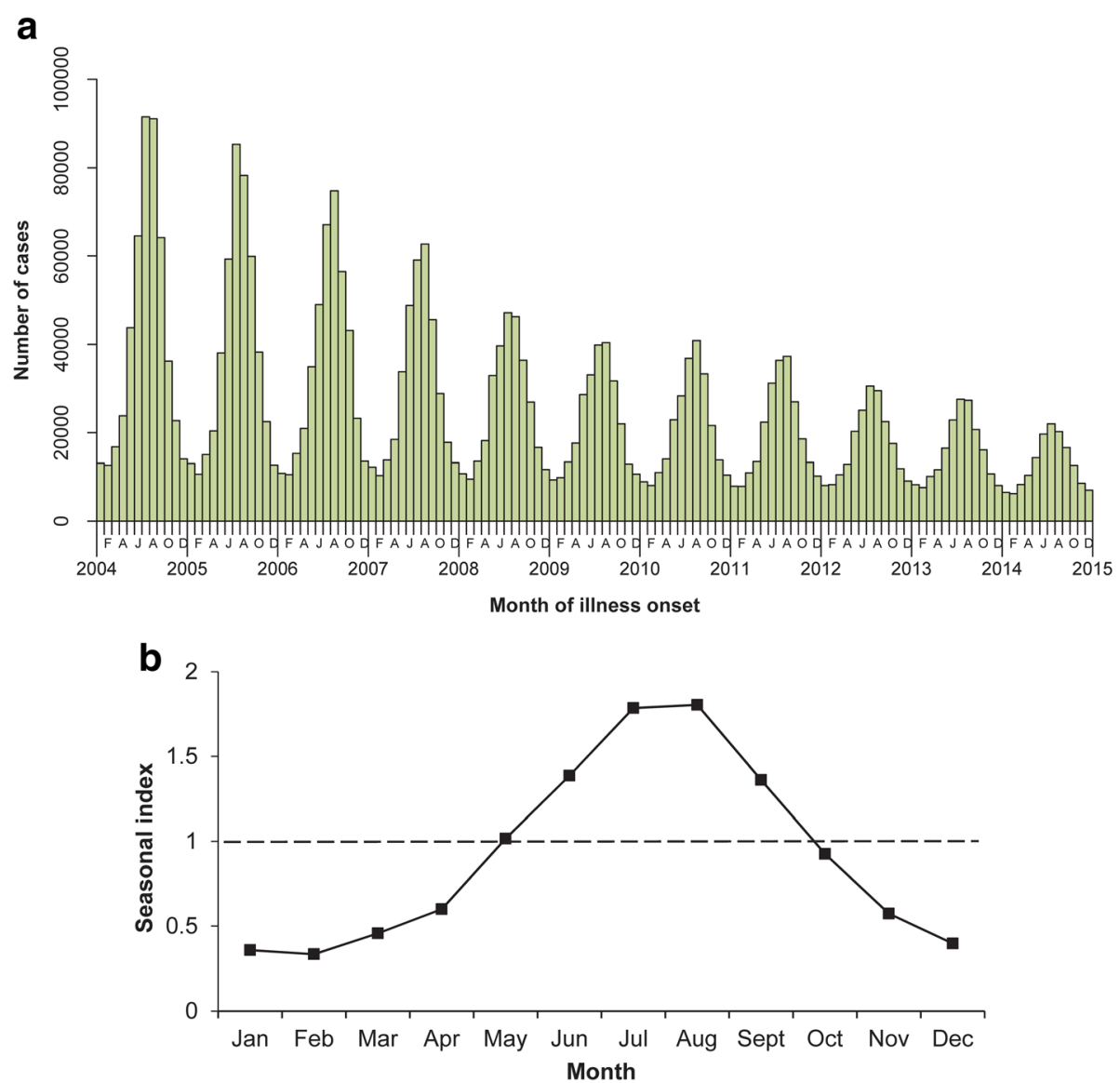

Fig. 3 Seasonal distribution of bacillary dysentery cases in China, 2004-2014. a The frequency distribution of bacillary dysentery cases by month of illness onset. $\mathbf{b}$ The seasonal index of bacillary dysentery. The seasonal index was calculated by month as the average case count for a given month divided by the average monthly case count during the entire 11-year time period, 2004-2014. No obvious seasonal pattern was expected if the seasonal index of each month was close to 1.0

against nalidixic acid (89.13\% of tested isolates resistant), followed by ampicillin (88.90\%), tetracycline (88.43\%), and sulfamethoxazole $(82.92 \%)$. The proportion of isolates resistant to nalidixic acid, ampicillin, tetracycline, or sulfamethoxazole was consistently high during 20052014 (Fig. 6a). Furthermore, the percentage of isolates resistant to ciprofloxacin and cefotaxime increased from 8.53 and $7.87 \%$ in 2005 to 44.65 and $29.94 \%$ in 2014 ( $Z$ $=18.31$ and 10.22, $P<0.01$, by Cochran-Armitage trend test), respectively (Fig. 6b).

\section{Discussion}

This study explored the morbidity, mortality, sex-specific annual incidence, and geographic and temporal distribution of bacillary dysentery in China using the surveillance dataset from the NIDRS during 2004-2014. In addition, the dominant Shigella species that cause bacillary dysentery along with their resistance to common antimicrobial drugs were analyzed using samples collected at sentinel hospitals during 2005-2014.
The incidence rate of bacillary dysentery during 2004-2014 in China showed an overall decline. The lowest rate (11.24 per 100,000 person-years) was observed in 2014, which was less than one third of the incidence rate in 2004 (38.03 per 100,000 person-years). A number of factors may have contributed to the observed decline, including rapid economic development, improvement of water supply systems, improved sanitary facilities, and a rising awareness of the importance of sanitation [16]. However, the number of cases as well as the incidence rate of bacillary dysentery in China remains higher than that in many developed countries, such as the USA, Australia, England and France, where the incidence of bacillary dysentery reported was 1.8-6.5 cases per 100,000 person-years [7]. Some regions in northwest China (including Tibet, Xinjiang, Gansu, Qinghai, and Ningxia) and in northern China (including Beijing and Tianjin) consistently showed higher morbidity than other regions in China during the study period. The high incidence rates of bacillary dysentery in Tibet, Xinjiang, Qinghai, Gansu, 


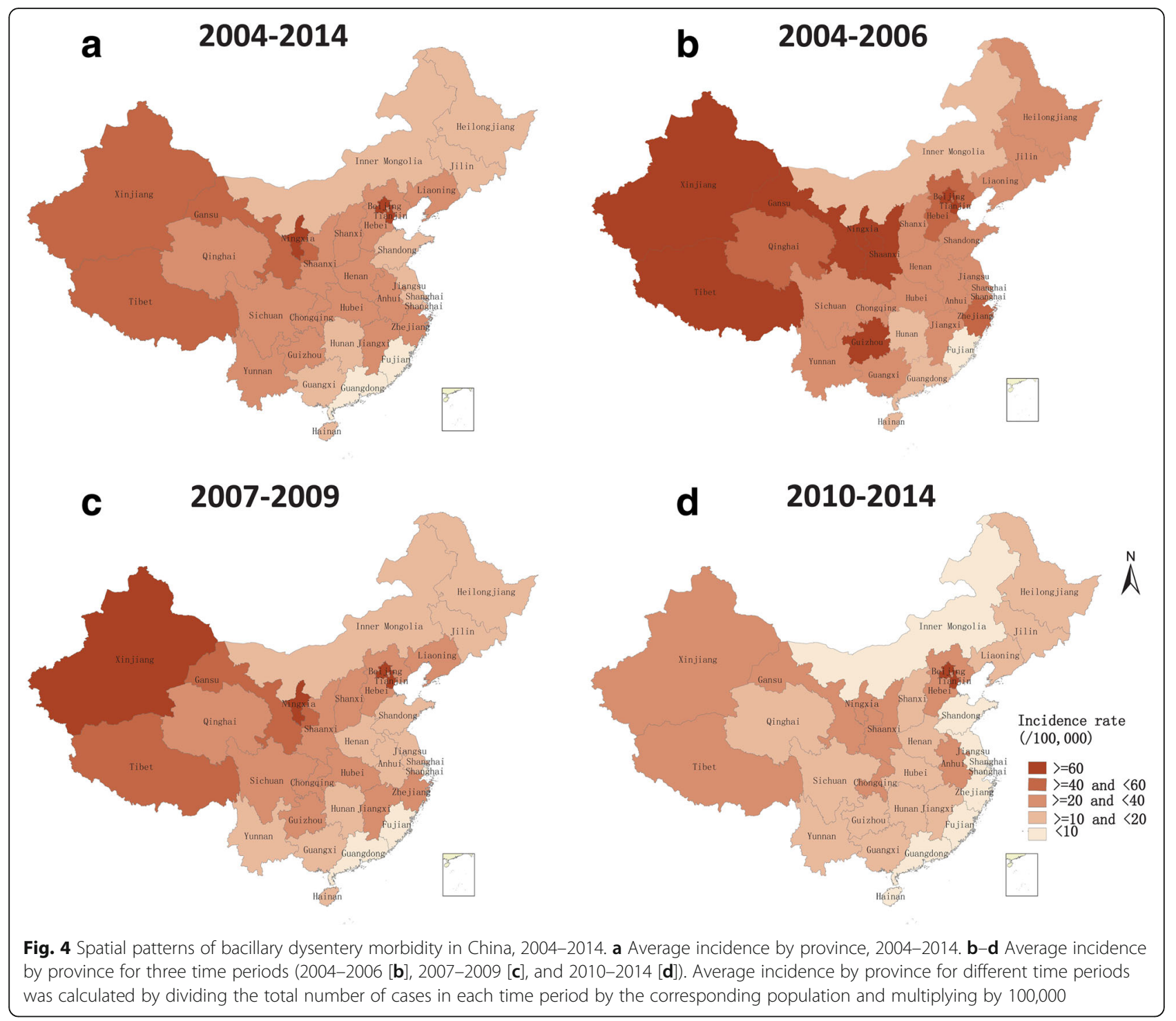

and Ningxia are consistent with their relatively low Gross Domestic Product [14]. These results indicate that economic development, which is tightly associated with the condition and accessibility of health facilities and healthcare resources, is potentially one of the major factors determining the occurrence of bacillary dysentery [17]. The high incidence rate observed in Beijing and Tianjin could potentially be explained by the high population density and good case ascertainment rates in these two regions [18]. These two regions are also known for having a large transient population who mostly live in suburban areas without proper sanitation and access to clean water [19].

In this study, bacillary dysentery was observed in all age groups, but individuals $<5$ years of age and individuals $\geq 60$ years of age had higher incidence rates of bacillary dysentery in China. Unlike in other countries, the highest incidence rate was observed in infants $<1$ year of age [7, 20-23]. The finding suggests that additional studies are needed to statistically or mechanistically characterize risk factors of bacillary dysentery associated with breastfeeding infants in China. Children $<5$ years of age are likely susceptible to bacillary dysentery because of low immune function, lack of previous exposure, and a higher chance of exposure to a Shigellacontaminated environment through play-related activities $[24,25]$. The difference in incidence between male and female individuals, especially those $<5$ years of age may be because male individuals are generally more active than female individuals, and thus have more opportunities to be exposed to environments containing bacteria [19]. Over the study period, we also observed a decrease in the proportion of bacillary dysentery cases identified in individuals 5-19 years of age and a corresponding increase in 

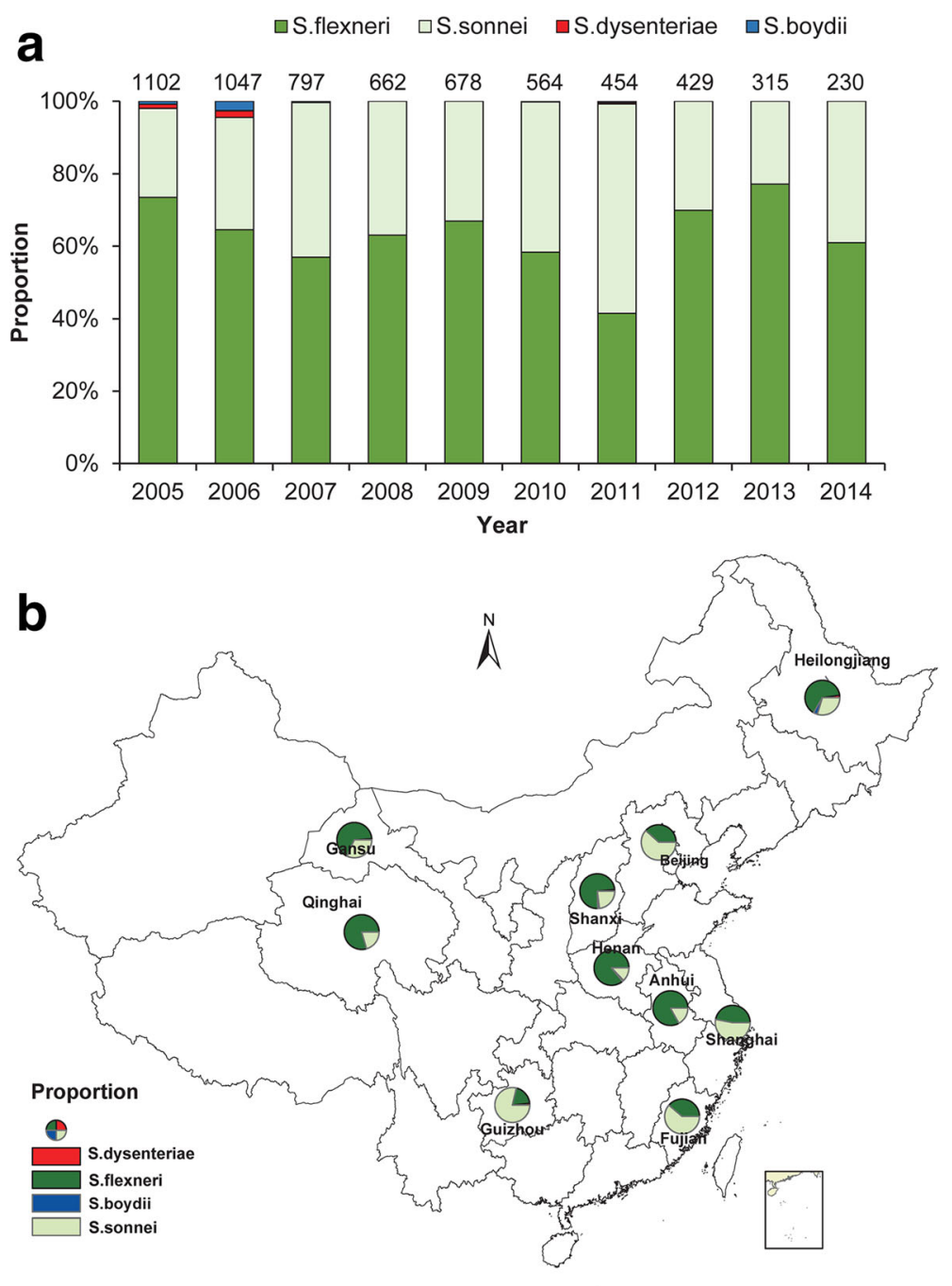

Fig. 5 Proportion of Shigella spp. isolated in laboratory-confirmed cases of bacillary dysentery by year and province in sentinel hospitals, 2005-2014. a The temporal distribution of the proportion by Shigella spp. $\mathbf{b}$ The geographical distribution of the proportion by Shigella spp.

Table 1 Antimicrobial resistance of Shigella isolates obtained in sentinel hospitals in China, 2005-2014

\begin{tabular}{lll}
\hline Antibiotic & $\begin{array}{l}\text { Number of isolates } \\
\text { tested }\end{array}$ & $\begin{array}{l}\text { Percentage of resistant } \\
\text { isolates (\%) }\end{array}$ \\
\hline Nalidixic acid & 4178 & 89.13 \\
Ampicillin & 4335 & 88.90 \\
Tetracycline & 4247 & 88.43 \\
Sulfamethoxazole & 4223 & 82.92 \\
Amoxicillin & 4060 & 53.17 \\
Cephalothin & 3952 & 34.82 \\
Gentamicin & 4254 & 34.28 \\
Ciprofloxacin & 3583 & 22.10 \\
Cefotaxime & 4247 & 19.10 \\
\hline
\end{tabular}

the proportion of cases in individuals $\geq 60$ years of age. Such a shift in relative age-specific risk may be because the majority of individuals 5-19 years of age are school students. In the past decades, schools have strengthened preventive measures against bacillary dysentery by providing safe drinking water, safe food, and health education. All together, our findings suggest that preventive interventions to control bacillary dysentery should emphasize protection for individuals $<5$ years of age and individuals $\geq 60$ years of age. Within each year, incidence rates of bacillary dysentery generally peaked between June and September. Previous studies have demonstrated that temperature plays an important role in the seasonal fluctuation of the incidence of bacillary dysentery as variation in temperature can impact the survival and reproduction of Shigella bacteria [26-31]. 

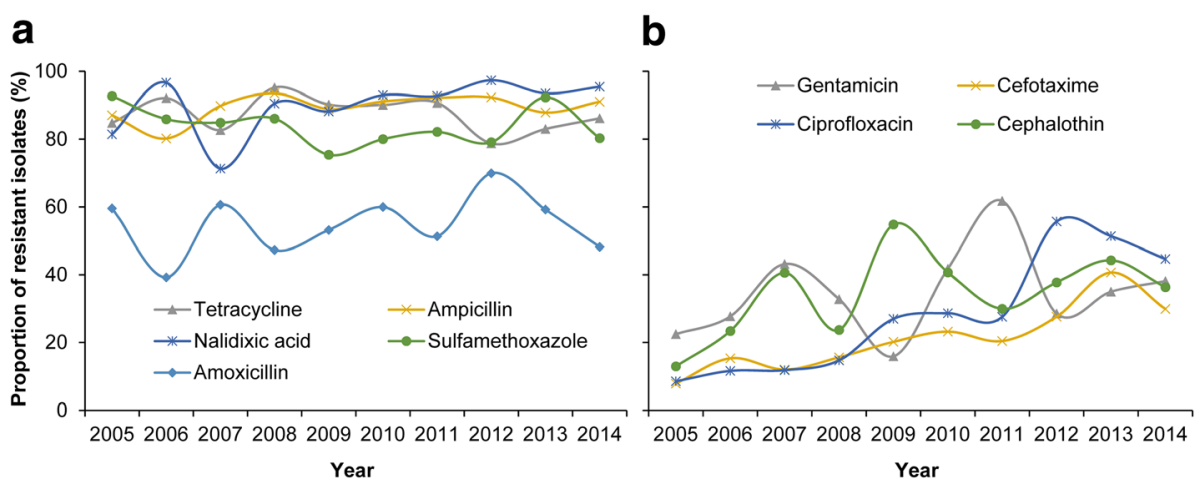

Fig. 6 Proportion of Shigella isolates resistant to different antimicrobial drugs in China, 2005-2014. a Proportion of Shigella isolates resistant to nalidixic acid, ampicillin, tetracycline, sulfamethoxazole, and amoxicillin. b Proportion of Shigella isolates resistant to Cephalothin, Gentamicin, Ciprofloxacin, and Cefotaxime. The proportion was calculated by dividing the number of isolates resistant to each antibiotic by all tested isolates

The distribution of species and serotypes of Shigella is dynamic over time and place. Based on our findings, the species distribution of Shigella in China was similar to that of other developing countries such as Bangladesh, Peru, and India [32-34]. Nonetheless, the distribution of Shigella species varied among different regions in China. Similar to developed countries, S. sonnei was the dominant species observed in Beijing, Shanghai, Guizhou, and Fujian. Beijing and Shanghai have undergone rapid economic development in recent years and represent the most developed regions in China. Interestingly, the dominant Shigella species in Beijing before 2006 was $S$. flexneri [35]. The transition of the predominant Shigella species from S. flexneri to S.sonnei was also observed in other fast-developing countries such as Thailand, Brazil, and Turkey, which suggests that regional socioeconomic development might predict the distribution of Shigella species. However, Guizhou and Fujian are located in southern China and have different meteorological conditions with high relative humidity and temperature [36].

Effective antimicrobial therapy of bacillary dysentery can significantly relieve symptoms and shorten both the duration of infection and the excretion time of bacteria [37]. In recent years, antimicrobial resistant Shigella strains have been commonly identified, threatening and complicating effective treatment of bacillary dysentery. Our study shows that Shigella isolated in China tend to have a higher resistance to nalidixic acid, ampicillin, tetracycline, and sulfamethoxazole than that found in many other countries $[38,39]$. These antibiotics were previously used as first-line treatments and are still commonly prescribed to treat bacillary dysentery. From 2005 to 2014, there was also a gradual increase in the prevalence of Shigella bacteria resistant to cefotaxime and ciprofloxacin, which have become the first-line agents for treating bacillary dysentery in adults. Such increase in ciprofloxacin-resistant Shigella bacteria have also been observed in the United States [40, 41]. If introduced to populations in nursing homes or childcare settings, Shigella bacteria may spread rapidly and cause large protracted outbreaks. The fact that patterns of antimicrobial resistance of Shigella bacteria are continuously changing underscores the importance of continuous monitoring of antimicrobial susceptibility of Shigella bacteria and emphasizes how antimicrobial treatment of bacillary dysentery should be carried out according to antimicrobial susceptibility data.

This study has several limitations. First, most cases of bacillary dysentery with mild symptoms can be cured by oral antibiotics. Therefore, under-reporting may have occurred. Second, differences in health care seeking behaviours and access to health care may exist across different provinces, which may result in information bias. Third, this study is an analysis on routine surveillance data, and we were unable to collect detailed data of different Shigella species to examine the differences between them in terms of epidemic characteristics, clinical symptoms, and antimicrobial resistance.

\section{Conclusions}

Bacillary dysentery has a considerable and continuing disease burden in China. However, the incidence rate of bacillary dysentery has been decreasing, presumably due to socioeconomic development, implementation of sanitation facilities, and health education. Children $<5$ years of age, particularly infants $<1$ year of age, have a higher incidence rate of bacillary dysentery than individuals in other age groups. Different regions in China differ in their peak incidence time. The incidence rate of bacillary dysentery remains high in northwest China. Corresponding to an extensive use of antimicrobial drugs, the proportion of antimicrobial-resistant Shigella bacteria is increasing, threatening effective treatment of bacillary dysentery. Priority interventions should be delivered to populations in northwest China and individuals $<5$ years of age. Effective treatment of bacillary 


\section{dysentery must be guided by continuous monitoring of antibiotic resistance patterns.}

\section{Abbreviations}

ATCC: American Type Culture Collection; CDC: Centers for Disease Control and Prevention; CLSI: Clinical Laboratory Standards Institute; HPF: High power field; NIDRS: National Infectious Disease Information Reporting System

\section{Acknowledgements}

We are very thankful to the staff at the hospital, local health departments, local Centers for Disease Control and Prevention (CDC) and China CDC for their assistance in coordinating data collection. We thank Wu Shuyu, Barbara Mahon and Cindy Friedman from the CDC in United States for their comments on an earlier draft of this article. We also thank Yi Teing Cheung from Princeton University for editing the manuscript.

\section{Funding}

This study was supported by the National Health and Family Planning Commission. The funding bodies had no role in study design, data collection and analysis, preparation of the manuscript, or the decision to publish.

\section{Availability of data and materials}

All relevant data are within the paper.

\section{Authors' contributions}

QL conceived the analyses. ZC finalized the analysis and wrote the first draft of the manuscript. JZ, LR, JS, LL, LZ, ZL and HY assisted in data analysis and interpreted the findings. All authors contributed to refining the final version of the manuscript. All authors read and approved the final manuscript.

\section{Competing interests}

The authors declare that they have no competing interests.

\section{Consent for publication}

\section{Not applicable.}

\section{Ethics approval and consent to participate}

Bacillary dysentery is a notifiable diseases in China. According to China's law on the prevention and treatment of infectious diseases, personal identifiers should be collected for individual cases with diagnosis of a notifiable disease, for the purposes of public health surveillance and response. The National Health and Family Planning Commission of China decided that the collection of individual data for all notifiable diseases, including bacillary dysentery, according to the national surveillance protocol was part of an ongoing public health response and was thus exempt from institutional review board assessment.

The China CDC has strict regulations on how to protect patients' privacy. The National Center for Public Health Surveillance and Information Services at the China CDC is responsible for the management of all disease surveillance data, and it anonymized the individual bacillary dysentery data by deleting the personal identifiers (such as patient name, parent name, home address, and telephone number) before the China CDC co-authors of this article, in the Division of Infectious Disease, were given access to the surveillance data for the purposes of research. The co-authors of this article did not participate in de-identifying the data and do not have the personal identifiers of the bacillary dysentery cases. Data were also aggregated before analysis.

\section{Received: 5 February 2016 Accepted: 26 October 2016}

\section{Published online: 18 November 2016}

\section{References}

1. Nandy S, Dutta S, Ghosh S, Ganai A, Rajahamsan J, Theodore RB, Sheikh NK. Foodborne-associated Shigella sonnei, India, 2009 and 2010. Emerg Infect Dis. 2011;17:2072-4.

2. Nygren BL, Schilling KA, Blanton EM, Silk BJ, Cole DJ, Mintz ED. Foodborne outbreaks of shigellosis in the USA, 1998-2008. Epidemiol Infect. 2013;141:233-41.

3. Bhattacharya SK, Sur D, Mahalanabis D. Public health significance of shigellosis. Indian Pediatr. 2012;49:269-70.
4. Centers for Disease Control and Prevention (CDC). Notes from the field: Outbreak of infections caused by Shigella sonnei with decreased susceptibility to azithromycin—Los Angeles, California, 2012. MMWR Morb Mortal Wkly Rep. 2013;62:171.

5. He F, Han K, Liu L, Sun W, Zhang L, Zhu B, Ma H. Shigellosis outbreak associated with contaminated well water in a rural elementary school: Sichuan Province, China, June 7-16, 2009. PLoS One. 2012;7:e47239.

6. Niyogi SK. Shigellosis. J Microbiol. 2005;43:133-43.

7. Kotloff KL, Winickoff JP, Ivanoff B, Clemens JD, Swerdlow DL, Sansonetti PJ, Adak GK, Levine MM. Global burden of Shigella infections: implications for vaccine development and implementation of control strategies. Bull World Health Organ. 1999;77:651-66.

8. Pelczar Jr MJ, Chan ECS. Elements of microbiology. Tokyo: KogakushaMcGraw Hill; 1981. p. 13

9. Ashkenazi S, Levy I, Kazaronovski V, Samra Z. Growing antimicrobial resistance of Shigella isolates. J Antimicrob Chemother. 2003;51:427-9.

10. Yang W, Li Z, Lan Y, Wang J, Ma J, Jin L, Sun Q, Lv W, Lai S, Liao Y, et al. A nationwide web-based automated system for outbreak early detection and rapid response in China. West Pac Surveill Response J. 2011;2:10-5.

11. Ministry of Health of the People's Republic of China. Diagnostic Criteria for Bacillary and Amoebic Dysentery (WS 287-2008). 2008 (in Chinese).

12. National Health and Family Planning Commission. National Surveillance Project of Shigellosis. 2005. (in Chinese). http://www.nhfpc.gov.cn/zhuzhan/zcjd/ 201304/ac06031652644d308429d8a5a36ff443.shtml. Assessed 9 Apr 2015.

13. Clinical and Laboratory Standards Institute. Performance standards for antimicrobial susceptibility testing. approved standard, 14th edn, document M100-S14. 2004

14. National Bureau of Statistics of China. National census in China. (in Chinese). http://data.stats.gov.cn/easyquery.htm?cn = E0103. Assessed 9 Apr 2015.

15. Xiao D, Long Y, Wang S, Wu K, Xu D, Li H, Wang G, Yan Y. Epidemic distribution and variation of Plasmodium falciparum and Plasmodium vivax malaria in Hainan, China during 1995-2008. Am J Trop Med Hyg. 2012;87:646-54

16. Wang XY, Du L, Von Seidlein L, Xu ZY, Zhang YL, Hao ZY, Han OP, Ma JC, Lee HJ, Ali M, et al. Occurrence of shigellosis in the young and elderly in rural China: results of a 12-month population-based surveillance study. Am J Trop Med Hyg. 2005;73:416-22.

17. Ferrer SR, Strina A, Jesus SR, Ribeiro HC, Cairncross S, Rodrigues LC, Barreto ML. A hierarchical model for studying risk factors for childhood diarrhoea: a case-control study in a middle-income country. Int J Epidemiol. 2008;37:805-15

18. Gao TLG, Li X, Jia L, Liu Y, et al. Analysis about epidemic situation of dysentery near upon fourteen years in Beijing. Chin J Preve Med. 2007;41: 54-7 (in Chinese).

19. Xiao G, Xu C, Wang J, Yang D, Wang L. Spatial-temporal pattern and risk factor analysis of bacillary dysentery in the Beijing-Tianjin-Tangshan urban region of China. BMC Public Health. 2014;14:998.

20. Finkelstein $Y$, Moran O, Avitzur Y, Nussinovitch M, Harel L, Volovitz B, Amir J. Clinical dysentery in hospitalized children. Infection. 2002;30:132-5.

21. Edwards BH. Salmonella and Shigella species. Clin Lab Med. 1999;19:469-87. v.

22. Prado V, Lagos R, Nataro JP, San Martin O, Arellano C, Wang JY, Borczyk AA, Levine MM. Population-based study of the incidence of Shigella diarrhea and causative serotypes in Santiago, Chile. Pediatr Infect Dis J. 1999;18:500-5.

23. Yurdakok K, Sahin N, Ozmert E, Berkman E. Shigella gastroenteritis: clinical and epidemiological aspects, and antibiotic susceptibility. Acta Paediatr Jpn. 1997;39:681-4.

24. Ahmed K, Shakoori FR, Shakoori AR. Aetiology of shigellosis in northern Pakistan. J Health Popul Nutr. 2003:21:32-9.

25. Hossain MA, Albert MJ, Hasan KZ. Epidemiology of shigellosis in Teknaf, a coastal area of Bangladesh: a 10-year survey. Epidemiol Infect. 1990:105:41-9.

26. Gao L, Zhang Y, Ding G, Liu Q, Zhou M, Li X, Jiang B. Meteorological variables and bacillary dysentery cases in Changsha City, China. Am J Trop Med Hyg. 2014;90:697-704

27. Ma W, Sun X, Song Y, Tao F, Feng W, He Y, Zhao N, Yuan Z. Applied mixed generalized additive model to assess the effect of temperature on the incidence of bacillary dysentery and its forecast. PLoS One. 2013;8:e62122.

28. Zhang Y, Bi P, Hiller JE, Sun Y, Ryan P. Climate variations and bacillary dysentery in northern and southern cities of China. J Infect. 2007:55:194-200 
29. Checkley W, Epstein LD, Gilman RH, Figueroa D, Cama Rl, Patz JA, Black RE. Effect of El Nino and ambient temperature on hospital admissions for diarrhoeal diseases in Peruvian children. Lancet. 2000;355:442-50.

30. Guan P, Huang D, Guo J, Wang P, Zhou B. Bacillary dysentery and meteorological factors in northeastern China: a historical review based on classification and regression trees. Jpn J Infect Dis. 2008;61:356-60.

31. Huang D, Guan P, Guo J, Wang P, Zhou B. Investigating the effects of climate variations on bacillary dysentery incidence in northeast China using ridge regression and hierarchical cluster analysis. BMC Infect Dis. 2008;8:130.

32. Ram PK, Crump JA, Gupta SK, Miller MA, Mintz ED. Part II. Analysis of data gaps pertaining to Shigella infections in low and medium human development index countries, 1984-2005. Epidemiol Infect. 2008;136:577-603.

33. Fernandez-Prada CM, Venkatesan MM, Franco AA, Lanata CF, Sack RB, Hartman AB, Spira W. Molecular epidemiology of Shigella flexneri in a diarrhoea-endemic area of Lima, Peru. Epidemiol Infect. 2004;132:303-16.

34. Pazhani GP, Ramamurthy T, Mitra U, Bhattacharya SK, Niyogi SK. Species diversity and antimicrobial resistance of Shigella spp. isolated between 2001 and 2004 from hospitalized children with diarrhoea in Kolkata (Calcutta), India. Epidemiol Infect. 2005;133:1089-95.

35. Mao Y, Cui E, Bao C, Liu Z, Chen S, Zhang J, Wang H, Zhang C, Zou J, Klena $J D$, et al. Changing trends and serotype distribution of Shigella species in Beijing from 1994 to 2010. Gut Pathog. 2013;5:21.

36. Chompook P, Samosornsuk S, von Seidlein L, Jitsanguansuk S, Sirima N, Sudjai S, Mangjit P, Kim DR, Wheeler JG, Todd J, et al. Estimating the burden of shigellosis in Thailand: 36-month population-based surveillance study Bull World Health Organ. 2005:83:739-46.

37. Sur D, Ramamurthy T, Deen J, Bhattacharya SK. Shigellosis : challenges \& management issues. Indian J Med Res. 2004;120(5):454-62.

38. Ozmert EN, Ince OT, Orun E, Yalcin S, Yurdakok K, Gur D. Clinical characteristics and antibiotic resistance of Shigella gastroenteritis in Ankara, Turkey between 2003 and 2009, and comparison with previous reports. Int J Infect Dis. 2011;15:e849-53.

39. Pourakbari B, Mamishi S, Mashoori N, Mahboobi N, Ashtiani MH, Afsharpaiman S, Abedini M. Frequency and antimicrobial susceptibility of Shigella species isolated in Children Medical Center Hospital, Tehran, Iran, 2001-2006. Braz J Infect Dis. 2010;14:153-7.

40. Bowen A, Hurd J, Hoover C, Khachadourian Y, Traphagen E, Harvey E, Libby T, Ehlers S, Ongpin M, Norton JC, et al. Importation and domestic transmission of Shigella sonnei resistant to ciprofloxacin-United States, May 2014-February 2015. MMWR Morb Mortal Wkly Rep. 2015;64:318-20.

41. Folster JP, Pecic G, Bowen A, Rickert R, Carattoli A, Whichard JM. Decreased susceptibility to ciprofloxacin among Shigella isolates in the United States, 2006 to 2009. Antimicrob Agents Chemother. 2011;55:1758-60.

\section{Submit your next manuscript to BioMed Central and we will help you at every step:}

- We accept pre-submission inquiries

- Our selector tool helps you to find the most relevant journal

- We provide round the clock customer support

- Convenient online submission

- Thorough peer review

- Inclusion in PubMed and all major indexing services

- Maximum visibility for your research

Submit your manuscript at www.biomedcentral.com/submit

) Biomed Central 